\title{
THE ACCRETION DISK MODEL FOR THE FIRST HUNDRED DAYS OF THE OUTBURST EVOLUTION IN THE BLACK HOLE X-RAY NOVAE
}

\author{
S.-W. KIM ${ }^{1}$, J. C. WHEELER ${ }^{1}$, S. MINESHIGE ${ }^{2}$ \\ 1. Department of Astronomy, University of Texas at \\ Austin, RLM 15.308, Austin, TX 78712-1083, USA \\ 2. Department of Astronomy, Faculty of Science, Kyoto \\ University, Sakyo-ku, Kyoto 606-01, Japan
}

We present time-dependent, irradiated, accretion disk models for the black hole X-ray novae in the first hundred days of the dwarf nova-like outbursts, including the rise, precursor, maximum and the secondary reflare. This work is based on the disk instability model (Kim, Mineshige \& Wheeler 1996, Kim, Wheeler \& Mineshige 1996). The model is reasonably consistent with the observed optical light curves. The irradiators are the central hot region around the black hole, and the corona or chromosphere above the accretion disk. In addition, we include the time-dependent shadowing effect and consequent blocking of the outer portions of the disk from the central irradiator. We find the stagnation phenomenon whereby the disk stays in the intermediate temperature stage between the hot and cool state. This can explain the recently discovered optical precursor rise prior to the maximum light in Nova Sco 1994 (Bailyn et al. 1995: see Fig. 1). We suggest the secondary re-flare after the maximum is due to the coupled effects of the irradiation and stagnation. In the model, the stagnation phenomenon during the rise results from the partial ionization and molecular opacity. In addition, we find irradiation-induced stagnation during the decay phase, which is consistent with the observed secondary re-flare in $\mathrm{X}$-ray novae (see Fig. 1). In the overall evolution of model outbursts in the first hundred days, the outer disk is blocked from the irradiation and, in turn, the companion star may not be strongly irradiated. This suggests that there is no appreciable increase of mass transfer rate during the decay prior to the secondary re-flare, unlike the behaviour in the mass transfer burst models. 


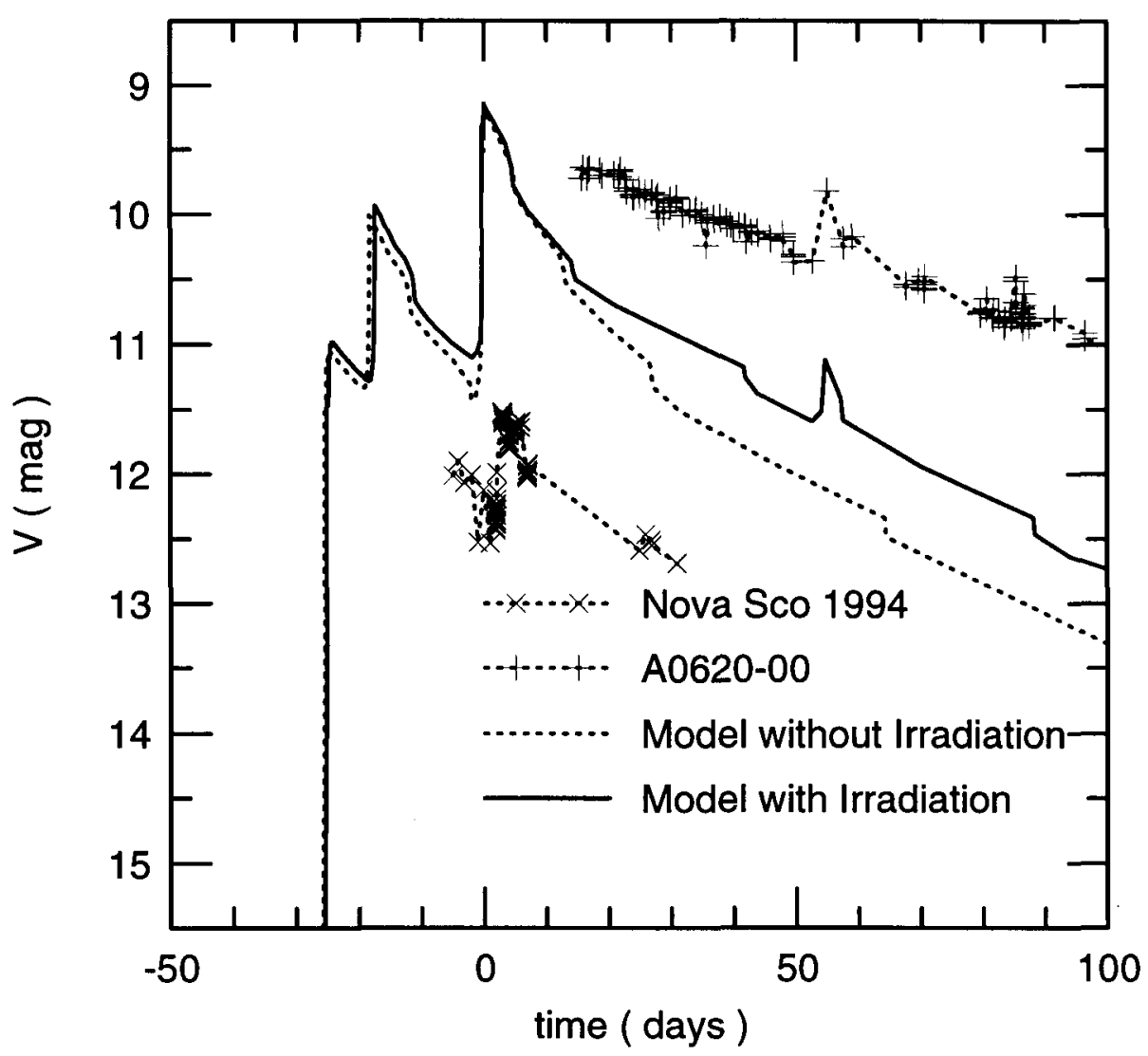

Figure 1. Model light curves for A0620-00 are presented for the case with no irradiation (dash line) and irradiation (solid line) along with the optical observations of A0620-00 (Webbink 1978) and Nova Sco 1994 (Bailyn et al. 1995). The data for A0620-00 and Nova Sco 1994 are plotted $1.5 \mathrm{mag}$ brighter and $2.5 \mathrm{mag}$ dimmer than observations, respectively. Note that Nova Sco 1994 revealed a secondary re-flare much sooner than that in $\mathrm{A} 0620-00$.

\section{References}

Bailyn, C. D., Orosz, J. A., Girard, T. M., et al., 1995, Nature, 374, 701 Kim, S.-W., Mineshige, S., Wheeler, J. C., 1996, to be submitted to ApJ Kim, S.-W., Wheeler, J. C., Mineshige, S. 1996, to be submitted to ApJ Webbink, R. F., 1978, University of Illinois, and private communication 